\title{
Estudio de prevalencia y letalidad neonatal en pacientes con anomalías congénitas seleccionadas con datos del Registro Nacional de Anomalías Congénitas de Argentina Study on the prevalence and neonatal lethality in patients with selected congenital anomalies as per the data of the National Registry of Congenital Anomalies of Argentina
}

\author{
Dra. María Paz Bidondo ${ }^{a}$, Dr. Boris Groisman ${ }^{,}$, Lic. Juan A. Gilia, , \\ Dra. Rosa Liascovich ${ }^{a}$ y Dr. Pablo Barbero ${ }^{a}$ \\ Colaboradores: Magíster Verónica Pingray y Miembros del Grupo de Trabajo \\ RENAC (veáse el Anexo en formato electrónico)
}

\section{RESUMEN}

Introducción. Las anomalías congénitas (AC), en Argentina, representan el $26 \%$ delas defunciones infantiles. La tasa de letalidad de AC mide el riesgo de morir entre los afectados.

Objetivos. Describir la prevalencia al nacer de un grupo de AC seleccionadas, estimar la tasa de letalidad neonatal y analizar su asociación con diferentes variables.

a. RENAC (Registro Nacional de Anomalías Congénitas), Centro Nacional de Genética Médica (CNGM), Administración Nacional de Laboratorios e Institutos de Salud (ANLIS).

b. Estudio Colaborativo Latinoamericano de Malformaciones Congénitas (ECLAMC) - CEMIC.

Correspondencia:

Dra. María Paz Bidondo, mariapazbidondo@ gmail.com

Financiamiento:

El trabajo ha sido parcialmente financiado por la Comisión Nacional Salud Investiga, a través de una beca CarrilloOñativia convocatoria 2012 y del Plan Nacer, Ministerio de Salud.

Conflicto de intereses: Ninguno que declarar.

Recibido: 2-12-2014

Aceptado: 12-3-2015
Población y métodos. El estudio se realizó con datos del Registro Nacional de Anomalías Congénitas. Se calcularon las prevalencias de encefalocele, espina bífida, gastrosquisis, onfalocele, hernia diafragmática, atresia de esófago, atresia intestinal o malformación anorrectal (período 2009-2013). La letalidad se evaluó a los 7 y a los 28 días de vida en los afectados de presentación aislada (año 2013). Se analizó la asociación con las variables sexo, edad gestacional, peso al nacer, detección ecográfica prenatal, porcentaje de necesidades básicas insatisfechas del departamento de residencia materna, región geográfica y nivel de complejidad del hospital de nacimiento.

Resultados. Gastrosquisis fue la AC de mayor prevalencia (8,53/10000 nacimientos) y hernia diafragmática, la de mayor tasa de letalidad neonatal $(66,67 \%)$. En el total de las AC seleccionadas, fue significativa la asociación entre mayor edad gestacional y sobrevida a los 7 días-OR: 0,81 (0,70-0,95)-y a los 28 días de vida -OR: 0,79 (IC 95\%: 0,68-0,91)-. Mayor porcentaje de necesidades básicas insatisfechas se asoció a mayor letalidad con hernia diafragmática-OR: 1,59 (IC 95\%: 1,30-1,95) - y con atresia intestinal o malformación anorrectal -OR: 16,00 (IC 95\%: 1,63-157,24)-.

Conclusiones. Laalta prevalencia degastrosquisis coincide con el aumento a nivel mundial. La prematurez y el alto porcentaje de necesidades básicas insatisfechas incrementaron el riesgo de morir en los afectados.

Palabras clave: mortalidad neonatal, anomalias congénitas, letalidad, mortalidad infantil, Argentina.

http:/ /dx.doi.org/10.5546/aap.2015.295

\section{INTRODUCCIÓN}

En Argentina, la transición epidemiológica ${ }^{1}$ condujo, en las últimas décadas, a una disminución de la mortalidad infantil (MI) y de sus dos componentes, la mortalidad neonatal (antes de los 28 días de vida) y la mortalidad posneonatal (entre los 28 días y el año de vida). A partir de la década del 70, la mortalidad neonatal ha aumentado su importancia relativa y ha superado al componente posneonatal. En el año 2012, hubo, en Argentina, 8227 defunciones infantiles y una tasa de MI de 11,1 por 1000 nacidos vivos. La mayor parte de las defunciones ocurrieron en el período neonatal, que representó el $67 \%$ de las defunciones. ${ }^{2}$

En este marco, las anomalías congénitas (AC), que corresponden al capítulo XVII (q00-q99) de la Clasificación Internacional de Enfermedades, ${ }^{3}$ incrementaron su importancia relativa. Actualmente, son la segunda causa de MI, pero superan como causa de muerte a los trastornos relacionados con la prematurez y el bajo peso al nacer. Las AC representan el $26 \%$ de las defunciones infantiles ${ }^{2}$ y las muertes se producen, de manera predominante, en el período neonatal. ${ }^{4}$ Sin embargo, el peso relativo de las AC en la MI es geográficamente heterogéneo y depende, en su mayoría, del control de las otras causas de muerte en las diferentes jurisdicciones. ${ }^{4}$ 
El riesgo de morir en los neonatos afectados con AC depende de distintos factores, tales como la propia gravedad de cada anomalía específica, las características clínicas de los afectados y el acceso a cuidados médico-quirúrgicos oportunos. El presente trabajo se concentra en un conjunto de AC seleccionadas: encefalocele, espina bífida, gastrosquisis, onfalocele, hernia diafragmática, atresia/estenosis de esófago con/sin fístula, atresia/estenosis intestinales y malformaciones anorrectales. Estas AC fueron elegidas teniendo en cuenta su impacto en la morbimortalidad, en la prevalencia y por ser reducibles a través de intervenciones médico-quirúrgicas. No existen, hasta la fecha, publicaciones referentes a la tasa de letalidad neonatal de afectados con AC en nuestro país.

Los objetivos del presente trabajo son describir la prevalencia al nacer de las AC seleccionadas, calcular las tasas de letalidad neonatal y neonatal temprana en recién nacidos afectados con dichas AC de presentación aislada y analizar su asociación con diferentes variables en las maternidades del Registro Nacional de Anomalías Congénitas de Argentina (RENAC) a fin de identificar factores condicionantes que pudieran ser objeto de futuras intervenciones.

\section{POBLACIÓN Y MÉTODOS}

La población objetivo del presente estudio correspondió a los nacimientos ocurridos en los hospitales participantes del RENAC, cuya cobertura alcanzó, en el año 2013, el 65\% de los nacimientos del sector público y el $38 \%$ de los nacimientos totales de Argentina. Este registro se inició en el año 2009 en 4 hospitales y fue incorporando, en forma progresiva, las principales maternidades del país. Durante el año 2013, las instituciones participantes del sector público fueron 122. El RENAC está integrado por una red de neonatólogos que recolectan los datos en los hospitales y una coordinación. Los neonatólogos registran a todos los recién nacidos vivos y los fetos muertos $>500$ gramos que presentan $\mathrm{AC}$ al nacer, describen las anomalías y completan otras variables siguiendo procedimientos estandarizados en un manual operativo y en un atlas. En cada institución, hay, al menos, dos responsables del RENAC que envían los datos. La coordinación revisa la calidad de las descripciones y codifica las AC (a cargo de médicos genetistas), analiza y difunde la información en reportes periódicos. ${ }^{5}$ La organización y funcionamiento del RENAC fue publicada previamente. ${ }^{6}$
El presente estudio constó de dos fases: de prevalencia y de letalidad. En la primera fase, se incluyeron todos los recién nacidos vivos y los fetos muertos $>500$ gramos que presentaron alguna de las AC seleccionadas y que fueron detectados por el RENAC entre el 1 de noviembre de 2009 y el 31 de diciembre de 2013. Los casos fueron clasificados según fueran recién nacidos vivos o fetos muertos y según se tratara de casos con AC aisladas, AC múltiples o sindrómicos. Los casos con AC aisladas se definen como aquellos que presentan una $\mathrm{AC}$ mayor única, o dos o más AC mayores que corresponden a una secuencia o se encuentran en la misma estructura corporal. Los casos con AC múltiples se definen como aquellos que presentan AC mayores que afectan estructuras corporales diferentes, no relacionadas, que corresponden a un patrón conocido (asociaciones) o no. Los casos sindrómicos se definen como aquellos que presentan una causa definida.

Se calcularon las prevalencias de las AC seleccionadas según la distribución de Poisson, con un intervalo de confianza del 95\%. Para cada una de las AC seleccionadas, se calculó el porcentaje de recién nacidos vivos y de fetos muertos, y el de casos con AC aisladas, AC múltiples y sindrómicos.

En la segunda fase, estudio de letalidad, se llevó a cabo el seguimiento hasta el primer mes de vida de los recién nacidos entre el 1 de enero y el 31 de diciembre de 2013, que presentaron las AC seleccionadas en forma aislada. El análisis de la letalidad solo se realizó con los datos del año 2013, ya que el número de maternidades incorporadas para ese período se consideró adecuado.

Se calculó la tasa de letalidad neonatal temprana (TLNTe; fallecimiento antes de los 7 días de vida) y la tasa de letalidad neonatal total (TLNTo; fallecimiento antes de los 28 días de vida). Dichas tasas se expresaron como porcentaje y se calcularon como el cociente entre el número de recién nacidos vivos afectados con cada AC y fallecidos en cada período (numerador) sobre el número total de recién nacidos vivos afectados con cada AC (denominador).

Para investigar los factores intervinientes en la TLNTe y en la TLNTo, se analizó su asociación con las siguientes variables. Sexo: femenino/ masculino. Edad gestacional (EG): analizada como variable continua. Peso al nacer (peso): analizado como variable continua. Detección prenatal ecográfica: sí/no; se definió como no detección cuando el Servicio de Neonatología no 
disponía de información prenatal de la AC por ausencia de ecografía o frente a ningún hallazgo patológico. Nivel de complejidad del hospital de nacimiento (Categorías IIIB vs. Categorías IIIA + II de la clasificación del Ministerio de Salud de la Nación). ${ }^{7}$ Región geográfica del hospital de nacimiento: Patagonia, Centro, Cuyo, Noroeste y Noreste. Porcentaje de población con necesidades básicas insatisfechas (\%NBI $)^{8}$ en el departamento de residencia materna: analizado como variable continua. La información sobre la variable peso se obtuvo en el $98,4 \%$ de los casos; edad gestacional, en el $98,8 \%$; sexo, en el 98,8\%; diagnóstico prenatal, en el 100\%; nivel de hospital, en el 96,6\%; y \%NBI, en el 98,7\%.

En un primer paso, el análisis de asociación se realizó agrupando a todos los recién nacidos con las AC seleccionadas. Como medida de asociación, para cada variable, se calculó el riesgo (OR) ajustado en un análisis multivariado de regresión logística. En un segundo paso, se analizó cada una de las AC y se evaluó la asociación mediante un análisis bivariado para estimar el riesgo (OR). Para evaluar la asociación entre el nivel del hospital y el diagnóstico prenatal, se realizó una prueba de Chi cuadrado ajustado utilizando el test de Mantel Haenszel para calcular el valor de $\mathrm{p}$ estratificando por tipo de malformación y tomando un nivel de significación estadística de 0,05 (error $\alpha=5 \%$ ). El software estadístico utilizado fue Stata 11.1.

\section{RESULTADOS}

Se observaron 2275 casos que presentaron las AC seleccionadas, en un total de 703325 nacimientos examinados entre el 1 de noviembre de 2009 y el 31 de diciembre de 2013. De estos casos, 95,64\% fueron recién nacidos vivos y $4,31 \%$ fueron fetos muertos; $66,24 \%$ de los casos presentaron AC aisladas, $26,29 \%$ presentaron AC múltiples y 7,47\% fueron casos sindrómicos. La AC de mayor prevalencia fue gastrosquisis (Tabla 1).

Para el estudio de letalidad, se detectaron, entre el 1 de enero y el 31 de diciembre de 2013, 528 recién nacidos vivos que presentaron en forma aislada alguna de las AC seleccionadas. En 471/528 $(89,20 \%)$, pudo obtenerse el dato de supervivencia hasta los 7 días de edad y, en 436/528 (82,58\%), se pudo continuar el seguimiento hasta los 28 días. Hernia diafragmática fue la anomalía con las mayores TLNTe $(57,38 \%)$ y TLNTo $(66,67 \%)$; espina bífida fue la que presentó la menor TLNTo $(2,50 \%)$ y no tuvo fallecidos antes de los 7 días de vida; las restantes AC presentaron valores intermedios (Tabla 2).

En relación con las variables estudiadas, se observaron los siguientes resultados para el total de los 528 afectados con las AC seleccionadas de presentación aislada: 394/510 $(77,25 \%)$ nacieron en hospitales de nivel IIIB; la razón de masculinidad fue $1,10(276 / 251)$; el peso medio fue 2667,10 gramos ( $\mathrm{DE}=678,40$ ); la edad

TABla 1. Prevalencia al nacer de las anomalías congénitas seleccionadas y distribución según su desenlace y su presentación clinica. Registro Nacional de Anomalías Congénitas, Argentina, 2009-2013

\begin{tabular}{|c|c|c|c|c|c|c|c|c|c|c|c|c|c|}
\hline \multirow[t]{3}{*}{$\begin{array}{l}\text { Anomalía } \\
\text { congénita }\end{array}$} & \multirow[t]{3}{*}{ Casos } & \multirow{3}{*}{$\begin{array}{c}\text { Prevalencia } \\
\text { x } 10000(\text { IC 95\%)* }\end{array}$} & \multicolumn{5}{|c|}{ Casos según el desenlace } & \multicolumn{6}{|c|}{$\begin{array}{c}\text { Casos según la presentación } \\
\text { clínica }\end{array}$} \\
\hline & & & \multicolumn{2}{|c|}{ Nacidos vivos } & \multicolumn{2}{|c|}{ Fetos muertos } & \multirow[t]{2}{*}{ NE } & \multicolumn{2}{|c|}{ Aislado } & \multicolumn{2}{|c|}{ Múltiple } & \multicolumn{2}{|c|}{ Síndrome } \\
\hline & & & $\mathbf{n}$ & $\%$ & n & $\%$ & & $\mathrm{n}$ & $\%$ & $\mathbf{n}$ & $\%$ & $\mathbf{n}$ & $\%$ \\
\hline Gastrosquisis & 600 & $8,53(7,86-9,24)$ & 573 & 95,50 & 27 & 4,50 & 0 & 546 & 91,00 & 52 & 8,67 & 2 & 0,33 \\
\hline Espina bífida & 389 & $5,53(4,99-6,11)$ & 372 & 95,87 & 16 & 4,12 & 1 & 305 & 78,41 & 72 & 18,51 & 12 & 3,08 \\
\hline Malformación anorrectal & 231 & $3,28(2,87-3,74)$ & 218 & 94,37 & 13 & 5,63 & 0 & 116 & 50,22 & 112 & 48,48 & 3 & 1,30 \\
\hline Atresia de esófago & 230 & $3,27(2,86-3,72)$ & 227 & 98,70 & 3 & 1,30 & 0 & 98 & 42,61 & 117 & 50,87 & 15 & 6,52 \\
\hline Hernia diafragmática & 225 & $3,20(2,79-3,65)$ & 219 & 97,33 & 6 & 2,67 & 0 & 164 & 72,89 & 54 & 24,00 & 7 & 3,11 \\
\hline $\begin{array}{l}\text { Atresia intestinal } \\
\text { (yeyuno-íleon) }\end{array}$ & 218 & $3,10(2,70-3,54)$ & 217 & 99,54 & 1 & 0,46 & 0 & 110 & 50,46 & 32 & 14,68 & 76 & 34,86 \\
\hline Onfalocele & 167 & $2,37(2,03-2,76)$ & 144 & 86,23 & 23 & 13,77 & 0 & 64 & 38,32 & 93 & 55,69 & 10 & 5,99 \\
\hline Atresia duodenal & 118 & $1,68(1,39-2,01)$ & 117 & 99,15 & 1 & 0,85 & 0 & 53 & 44,92 & 24 & 20,34 & 41 & 34,75 \\
\hline Encefalocele & 97 & $1,38(1,12-1,68)$ & 89 & 91,75 & 8 & 8,25 & 0 & 51 & 52,58 & 42 & 43,30 & 4 & 4,12 \\
\hline Total & 2275 & & 2176 & 95,64 & 98 & 4,31 & & 1507 & 66,24 & 598 & 26,29 & 170 & 7,47 \\
\hline
\end{tabular}

NE: no especificado; IC: intervalo de confianza.

* Prevalencia calculada sobre 703325 nacimientos en el total de casos (nacidos vivos y fetos muertos). 
gestacional media fue 36,90 semanas ( $\mathrm{DE}=2,43)$; el \%NBI medio fue $10,71 \%$, y $308 / 528$ (58,33\%) tuvieron diagnóstico prenatal. El porcentaje de detección prenatal para cada anomalía fue 88,89\% para encefalocele; $74,02 \%$ para gastrosquisis; $68,57 \%$ para espina bífida; $62,50 \%$ para onfalocele; $65,4 \%$ para hernia diafragmática; $25,6 \%$ para atresias esofágicas; $23,33 \%$ para atresias intestinales y malformaciones anorrectales. En los hospitales de nivel IIIB, el 65,52\% de los casos había tenido diagnóstico prenatal, mientras que, en los hospitales II o IIIA, solamente el $34,48 \%$ había sido diagnosticado prenatalmente. Se observó una asociación estadísticamente significativa entre la detección prenatal y el nivel del hospital de nacimiento $\left(\mathrm{chi}^{2}=26,59 ; \mathrm{p} \leq 0,001\right)$ y se estratificó por las 7 categorías de AC.

En el análisis que agrupó a todos los recién nacidos con las AC seleccionadas, se observó una asociación estadísticamente significativa entre la supervivencia de los afectados y la edad gestacional tanto en el período neonatal temprano
(OR ajustado 0,81; IC 95\%=0,70-0,95) como en el período neonatal total (OR ajustado 0,79; IC $95 \%=0,68-0,91)$. Asimismo, el incremento del $\%$ NBI del departamento de residencia materna mostró una tendencia a aumentar el riesgo de muerte en ambos períodos, aunque no resultó estadísticamente significativa. Se excluyó la variable detección prenatal por ser colineal con la variable nivel de hospital (Tabla 3).

El Noroeste argentino (NOA) fue la región con el mayor porcentaje de fallecidos en el período neonatal; sin embargo, las diferencias entre regiones no resultaron estadísticamente significativas $(\mathrm{p}=0,147)$ (Tabla 4$)$. El porcentaje de cobertura de nacimientos del subsector público fue para todas las regiones mayor del 50\%.

En el análisis llevado a cabo para las AC individualmente, se excluyó onfalocele y encefalocele por tener un número de casos insuficiente. El número de fallecidos en cada AC no permitió calcular los riesgos ajustados. Se observó una asociación estadísticamente

TABLA 2. Recién nacidos con anomalías congénitas seleccionadas de presentación aislada: porcentaje de seguimiento y fallecidos antes de los 7 y los 28 días de vida. Registro Nacional de Anomalías Congénitas, Argentina, 2013

\begin{tabular}{lccccc} 
AC & $\begin{array}{c}\text { Total de } \\
\text { afectados }\end{array}$ & $\begin{array}{c}\text { Seguimiento hasta } \\
\text { los 7 días de vida }\end{array}$ & $\begin{array}{c}\text { Seguimiento hasta } \\
\text { los 28 días de vida }\end{array}$ & $\begin{array}{c}\text { Fallecidos antes de } \\
\text { los 7 días de vida } \\
\text { (TLNTe) }\end{array}$ & $\begin{array}{c}\text { Fallecidos antes de } \\
\text { los 28 días de vida } \\
\text { (TLNTo) }\end{array}$ \\
\hline Gastrosquisis & 204 & $186 / 204(91,18 \%)$ & $173 / 204(84,80 \%)$ & $10 / 186(5,38 \%)$ & $18 / 173(10,40 \%)$ \\
Espina bífida & 105 & $92 / 105(87,62 \%)$ & $80 / 105(76,19 \%)$ & $0 / 92(0,00 \%)$ & $2 / 80(2,50 \%)$ \\
Atresias intestinales y MAR & 90 & $78 / 90(86,67 \%)$ & $72 / 90(80,00 \%)$ & $1 / 78(1,28 \%)$ & $3 / 72(4,17 \%)$ \\
Hernia diafragmática & 65 & $61 / 65(93,85 \%)$ & $60 / 65(92,31 \%)$ & $35 / 61(57,38 \%)$ & $40 / 60(66,67 \%)$ \\
Atresias esofágicas & 39 & $32 / 39(82,05 \%)$ & $32 / 39(82,05 \%)$ & $4 / 32(12,50 \%)$ & $8 / 32(25,0 \%)$ \\
Onfalocele & 16 & $14 / 16(87,50 \%)$ & $13 / 16(81,25 \%)$ & $3 / 14(21,43 \%)$ & $6 / 13(46,15 \%)$ \\
Encefalocele & 9 & $8 / 9(88,89 \%)$ & $6 / 9(66,67 \%)$ & $1 / 8(12,50 \%)$ & $2 / 6(33,33 \%)$ \\
Total & 528 & $471 / 528(89,20 \%)$ & $436 / 528(82,58 \%)$ & $54 / 471(11,46 \%)$ & $79 / 436(18,12 \%)$ \\
\hline
\end{tabular}

AC: anomalías congénitas; TLNTe: tasa de letalidad neonatal temprana; TLNTo: tasa de letalidad neonatal total;

MAR: malformación anorrectal.

TABLA 3. Riesgo de fallecer en los períodos, neonatal temprano y neonatal total, en recién nacidos con anomalías seleccionadas, según diferentes variables. Registro Nacional de Anomalías Congénitas, Argentina, 2013

\begin{tabular}{|c|c|c|}
\hline \multirow[t]{2}{*}{ Variables } & \multicolumn{2}{|c|}{ Fallecimiento } \\
\hline & $\begin{array}{c}\text { Antes de los } 7 \text { días } \\
\text { de vida OR (IC 95\%) }\end{array}$ & $\begin{array}{l}\text { Antes de los } 28 \text { días } \\
\text { de vida OR (IC 95\%) }\end{array}$ \\
\hline$<$ Peso al nacer * & $1,00(0,99-1,00)$ & $1,00(0,99-1,00)$ \\
\hline$>$ Edad gestacional * & $0,81(0,70-0,95)$ & $0,79(0,68-0,91)$ \\
\hline Sexo femenino & $0,55(0,30-1,02)$ & $0,60(0,35-1,01)$ \\
\hline$>\%$ NBI del departamento de residencia materna & $1,05(1,00-1,10)$ & $1,05(1,00-1,10)$ \\
\hline Bajo nivel de complejidad del hospital de nacimiento & $0,77(0,36-1,68)$ & $0,58(0,29-1,20)$ \\
\hline
\end{tabular}

\%NBI: porcentaje de necesidades básicas insatisfechas; OR: razón de odds; IC: intervalo de confianza.

* Variables analizadas en forma continua. 
significativa entre la menor supervivencia y el incremento del \%NBI para hernia diafragmática y atresia intestinal y anorrectal. Asimismo, se encontró un riesgo mayor de 5 para bajo peso y nacimiento pretérmino en los casos con atresia esofágica y espina bífida, aunque estos resultados no fueron estadísticamente significativos (Tabla 5).

\section{DISCUSIÓN}

El presente trabajo constituye el primer estudio realizado en Argentina orientado a conocer la prevalencia y la letalidad de AC seleccionadas, con datos de alta cobertura provenientes de las 24 jurisdicciones del país. Tanto las prevalencias observadas como la proporción de casos aislados, múltiples y sindrómicos, según la categoría de $A C$, se encuentran dentro de los valores esperados de la literatura internacional. Cabe destacar, sin embargo, la alta frecuencia de gastrosquisis. Esta entidad presenta una frecuencia en aumento a nivel mundial. ${ }^{9}$ Esta situación ha sido fuertemente asociada con edades maternas jóvenes $y$, en menor medida, con otros factores, entre ellos: infecciones genitourinarias en el período periconcepcional, bajo índice de masa corporal, bajo nivel socioeconómico, consumo de tabaco, alcohol y drogas ilícitas. ${ }^{9-11}$

La tasa de detección ecográfica prenatal observada en los casos de encefalocele, espina bífida, gastrosquisis, hernia diafragmática y

TABLA 4. Número de casos fallecidos con anomalías congénitas seleccionadas aisladas y tasa de letalidad neonatal total según la región geográfica del hospital de nacimiento. Registro Nacional de Anomalías Congénitas, Argentina, 2013

\begin{tabular}{|c|c|c|c|c|c|c|c|c|c|}
\hline Región & Gastrosquisis & $\begin{array}{l}\text { Espina } \\
\text { bífida }\end{array}$ & $\begin{array}{c}\text { Atresias } \\
\text { intestinales } \\
\text { y MAR }\end{array}$ & $\begin{array}{c}\text { Hernia } \\
\text { diafragmática }\end{array}$ & $\begin{array}{c}\text { Atresia } \\
\text { esofágica }\end{array}$ & Onfalocele & Encefalocele & $\begin{array}{l}\mathrm{N} \text { fallecidos/ } \\
\mathrm{N} \text { afectados }\end{array}$ & $\begin{array}{l}\text { TLNTo } \\
\text { (IC 95\%) }\end{array}$ \\
\hline Patagoni & 0 & 0 & 0 & 2 & 0 & 0 & 0 & $2 / 26$ & $\begin{array}{c}7,69 \% \\
(0,93-27,80)\end{array}$ \\
\hline Centro & 11 & 1 & 0 & 19 & 4 & 6 & 2 & $43 / 249$ & $\begin{array}{c}17,27 \% \\
(12,50-23,27)\end{array}$ \\
\hline Cuyo & 0 & 0 & 0 & 5 & 0 & 0 & 0 & $5 / 33$ & $\begin{array}{c}15,15 \% \\
(5,00-35,36)\end{array}$ \\
\hline NOA & 3 & 1 & 3 & 12 & 3 & 0 & 0 & $22 / 80$ & $\begin{array}{c}27,50 \% \\
(17,23-41,64)\end{array}$ \\
\hline NEA & 4 & 0 & 0 & 2 & 1 & 0 & 0 & $7 / 48$ & $\begin{array}{c}14,58 \% \\
(5,86-30,05)\end{array}$ \\
\hline Total & 18 & 2 & 3 & 40 & 8 & 6 & 2 & $79 / 436$ & $\begin{array}{c}18,12 \% \\
(14,35-22,58)\end{array}$ \\
\hline
\end{tabular}

Región: Patagonia (Chubut, La Pampa, Neuquén, Río Negro, Santa Cruz, Tierra del Fuego); Centro (CABA, Buenos Aires, Córdoba, Entre Ríos, Santa Fe); Cuyo (La Rioja, Mendoza, San Juan y San Luis); NOA (Catamarca, Jujuy, Salta, Santiago del Estero y Tucumán); NEA (Corrientes, Chaco, Formosa y Misiones).

IC: intervalo de confianza; MAR: malformación anorrectal.

TABLA 5. Riesgo de fallecer antes de los 28 días en recién nacidos con anomalías seleccionadas analizadas individualmente, según las diferentes variables. Registro Nacional de Anomalías Congénitas, Argentina, 2013

\begin{tabular}{|c|c|c|c|c|c|}
\hline \multirow[t]{2}{*}{ Variables } & \multicolumn{5}{|c|}{ Anomalías aisladas OR (IC 95\%) } \\
\hline & $\begin{array}{c}\text { Hernia } \\
\text { diafragmática }\end{array}$ & $\begin{array}{c}\text { Atresia } \\
\text { esofágica }\end{array}$ & Gastrosquisis & $\begin{array}{l}\text { Espina } \\
\text { bífida }\end{array}$ & $\begin{array}{c}\text { Atresias intestinales } \\
\text { y MAR }\end{array}$ \\
\hline$<$ Peso al nacer* & $1,05(0,70-1,60)$ & $5,44(0,76-39,25)$ & $1,56(0,54-4,51)$ & $5,58(0,37-83,32)$ & 0,00 \\
\hline$>$ Edad gestacional* & $0,95(0,61-1,48)$ & $5,44(0,76-39,25)$ & $1,72(0,68-4,37)$ & $6,18(0,42-91,77)$ & 0,00 \\
\hline Sexo femenino & $0,77(0,51-1,17)$ & $0,95(0,28-3,27)$ & $1,06(0,44-2,54)$ & $1,05(0,07-16,23)$ & $\begin{array}{c}2,65 \\
(0,25-26,87)\end{array}$ \\
\hline $\begin{array}{l}>\% \text { NBI del departamento } \\
\text { de residencia materna }\end{array}$ & $1,59(1,30-1,95)$ & $1,80(0,50-6,51)$ & $1,45(0,46-4,58)$ & 0,00 & $\begin{array}{c}16,00 \\
(1,63-157,24)\end{array}$ \\
\hline $\begin{array}{l}\text { Bajo nivel de complejidad } \\
\text { del hospital de nacimiento }\end{array}$ & $0,86(0,53-1,38)$ & $0,41(0,06-2,84)$ & $0,38(0,05-2,72)$ & $2,55(0,17-38,93)$ & $1,69(0,16-17,43)$ \\
\hline
\end{tabular}

\%NBI: porcentaje de necesidades básicas insatisfechas; OR: razón de odds; IC: intervalo de confianza;

MAR: malformación anorrectal.

* Variables analizadas en forma continua. 
onfalocele fue más baja que las descritas en otros estudios. ${ }^{12,13}$ Los casos de atresias intestinales y esofágicas fueron las AC con menor tasa de detección, posiblemente porque los hallazgos ecográficos son indirectos. ${ }^{14,15}$

En el seguimiento de recién nacidos afectados, se logró recuperar más del $80 \%$ de los casos. Hernia diafragmática fue la AC que presentó la mayor tasa de letalidad, y la mayor parte de los fallecimientos se concentró en el período neonatal temprano. Esta anomalía es clasificada como "difícilmente reducible" según los criterios del Ministerio de Salud de la Nación, ${ }^{16}$ por lo tanto, los resultados observados son coincidentes con esta categorización. La alta tasa de letalidad de esta anomalía pudo ser el motivo del mayor porcentaje de fallecidos en la región del NOA.

Espina bífida fue la única AC sin fallecidos antes de los 7 días de vida y con menor TLNTo, lo cual es coincidente con los trabajos de seguimiento que han reportado la mayor parte de las muertes luego del año de vida. ${ }^{17,18}$

Las tasas de letalidad neonatal en los casos de atresia intestinal y atresia anorrectal observadas en nuestro estudio son semejantes a las reportadas en otros estudios. ${ }^{19}$ La letalidad neonatal en gastrosquisis, onfalocele y atresia esofágica observada en otras investigaciones ha estado, en general, por debajo de las cifras observadas en nuestro estudio. ${ }^{20-22}$ Concordante con nuestro estudio, la prematurez ha sido asociada en varios trabajos a una mayor tasa de mortalidad en afectados con las AC seleccionadas. ${ }^{18,20,23-25} \mathrm{Sin}$ embargo, algunas investigaciones realizadas en pacientes con gastrosquisis han mostrado que las cesáreas electivas pretérmino evitaron la aparición de daño inflamatorio intestinal y mejoraron el funcionamiento enteral por presentar menor tiempo de exposición de las asas al meconio del líquido amniótico. ${ }^{26,27}$ Otros trabajos encontraron resultados opuestos a esta última aseveración..$^{28,29}$

En nuestro estudio, si bien la asociación no fue estadísticamente significativa, los resultados mostraron una tendencia que correlacionó la menor supervivencia a medida que disminuyó el peso al nacer cuando se analizó específicamente atresia de esófago y mielomeningocele. En la bibliografía, se ha referido que el bajo peso es un factor de riesgo para la supervivencia de los recién nacidos afectados con AC. ${ }^{18,20,25,30}$ En la atresia esofágica, el peso del paciente es un criterio internacionalmente establecido como factor pronóstico de mortalidad para la evaluación prequirúrgica. ${ }^{31}$
En relación con el análisis de indicadores socioeconómicos desfavorables y AC, Pawluk y colaboradores ${ }^{32}$ encontraron una asociación entre la pobreza (medida por índices socioeconómicos familiares y regionales) y la prevalencia al nacer de comunicación interventricular (CIV) y de fisuras orales. Por su parte, Vrijheid y cols. observaron una asociación positiva entre madres de bajo nivel socioeconómico y recién nacidos con cardiopatías septales y anomalías digestivas, de causa no cromosómica. ${ }^{33}$ Bronberg y colaboradores no encontraron una asociación entre la tasa de mortalidad específica por AC y los departamentos de residencia materna socioeconómicamente desfavorecidos. ${ }^{4,34}$ En nuestro estudio, el incremento del \%NBI se asoció significativamente con el riesgo de morir, de modo específico, en los casos con hernia diafragmática y atresias intestinales/anorrectales. Este resultado podría estar indicando que los departamentos más empobrecidos corresponden a los lugares con mayores dificultades para el cuidado perinatal. Consistentemente con nuestros hallazgos, Smith y colaboradores encontraron un mayor riesgo de morir en neonatos con AC aisladas cuyas madres tenían mayor privación económica. ${ }^{35}$

La utilización de las tasas de letalidad estimadas puede servir como un indicador para evaluar el impacto de intervenciones sanitarias y como insumo para organizar redes de derivación prenatal o neonatal inmediata de los recién nacidos con AC. Recientemente, el Ministerio de Salud definió el denominado Paquete Perinatal de Alta Complejidad, que es uno de los ejes priorizados para la implementación del Programa SUMAR a partir del año 2013. Este conjunto de prestaciones incluye las AC analizadas en este estudio y reviste un carácter relevante, ya que fue seleccionado por su capacidad de impactar significativamente en la reducción del núcleo duro de la mortalidad infantil.

Una fortaleza del presente estudio es que incluyó recién nacidos de las principales maternidades del sector público de las 24 jurisdicciones del país, con una alta cobertura de nacimientos.

Una de las debilidades es la pérdida de seguimiento de recién nacidos afectados. Otra limitación es que, para el análisis de cada AC por separado, el tamaño muestral no permitió estimar el riesgo ajustado de cada AC en ambos períodos. Además, no puede descartarse el efecto de la llamada falacia ecológica para la variable \%NBI, 
al no disponer de datos de nivel individual sobre la condición socioeconómica de las madres de los afectados. Finalmente, si bien la cobertura de nacimientos en cada región fue mayor del 50\%, esta fue heterogénea.

\section{CONCLUSIÓN}

Las prevalencias de las AC estudiadas se encontraron dentro de los valores esperados. Gastrosquisis fue la AC de mayor prevalencia y hernia diafragmática, la de mayor tasa de letalidad. La prematurez y la privación socioeconómica fueron las variables que incrementaron el riesgo de morir en los afectados.

\section{REFERENCIAS}

1. Frenk J, Frejka T, Bobadilla JL, Stern C, et al. La transición epidemiológica en América Latina. Bol Oficina Sanit Panam 1991;111(6):485-96.

2. Ministerio de Salud de la Nación Argentina. Dirección de Estadísticas e Información de Salud. Estadísticas vitales. Información básica - Año 2012. Buenos Aires, 2013. [Acceso: 1 demarzo de 2014]. Disponibleen:http:/ /www.deis.gov. ar/publicaciones/archivos/Serie5Nro56.pdf.

3. The Royal College of Paediatrics and Child Health Classification - Paediatric Adaptation of ICD-10. European Surveillance of Congenital Anomalies. 2008. [Acceso: 16 de marzo de 2015]. Disponible en: http://www.eurocatnetwork.eu/content/EUROCAT-Q-Chapter-2008.pdf.

4. Bronberg R, Alfaro E, Chaves E, Dipierri J. Mortalidad infantil por malformaciones congénitas en Argentina: análisis del quinquenio 2002-2006. Arch Argent Pediatr 2009;107(3):203-11.

5. Registro Nacional de Anomalías Congénitas de Argentina. Reporte anual RENAC 2014. Análisis epidemiológico de las anomalías congénitas en recién nacidos registradas en el año 2013 en la República Argentina. Buenos Aires: Ministerio Salud de la Nación, 2014

6. Groisman B, Bidondo MP, Barbero P, Gili JA, etal. RENAC: Registro Nacional de Anomalías Congénitas de Argentina. Arch Argent Pediatr 2013;111(6):484-94.

7. Resolución 306/2002. Norma de organización y funcionamiento de los servicios de neonatología y cuidados intensivos neonatales. Ministerio de Salud de la Nación. Ciudad de Buenos Aires, Argentina, 23 de mayo de 2002.

8. Instituto Nacional de Estadísticas y Censos. Base de datos del INDEC, Censo Nacional de Población, Hogares y Viviendas 2010, procesado con Redatam+SP. Resultados básicos hogares al menos un indicador NBI. Buenos Aires, 2014. [Acceso: 2 de abril de 2014]. Disponible en: http:/ /200.51.91.245/argbin/RpWebEngine.exe/Portal Action? \&MODE=MAIN\&BASE=CPV2010B\&MAIN=We bServerMain.inl.

9. Castilla EE, Mastroiacovo P, Orioli IM. Gastroschisis: international epidemiology and public health perspectives. Am J Med Genet C Semin Med Genet 2008;148C(3):162-79.

10. Feldkamp ML, Reefhuis J, Kucik J, Krikov S, et al. Casecontrol study of self reported genitourinary infections and risk of gastroschisis: findings from the national birth defects prevention study, 1997-2003. BMJ 2008;336(7658):1420-3.

11. Rasmussen SA, Frías JL. Non-genetic risk factors for gastroschisis. Am J Med Genet C Semin Med Genet 2008;148C(3):199-212.
12. Boyd PA, Rounding C, Kurinczuk JJ. Third Report of the Congenital Anomaly Register for Oxfordshire, Berkshire and Buckinghamshire(CAROBB) Births 2005-2010. Oxford: National Perinatal Epidemiology Unit, 2012.

13. Campaña H, Ermini M, Aiello HA, Krupitzki H, et al. Prenatal sonographic detection of birth defect in 18 hospitals from South America. J Ultrasound Med 2010;29(2):203-12.

14. Calisti A, Olivieri C, Coletta R, Briganti V, et al. Jejunoileal atresia: factors affecting the outcome and long term sequelae. J Clin Neonatol 2012;1(1):38-41.

15. Houben $\mathrm{CH}$, Curry JI. Current status of prenatal diagnosis, operative management and outcome of esophageal atresia / tracheo-esophageal fistula. Prenat Diagn 2008;28(7):667-75.

16. Argentina. Ministerio de Salud de la Nación, Sociedad Argentina de Pediatría. Mortalidad Infantil según Criterios de Reducibilidad. Tercera revisión. Sistema Estadístico de Salud. Serie 3. Número 56. Buenos Aires, 2014. [Acceso: 20 de enero de 2015]. Disponible en: http:/ / www.msal. gov.ar/images/stories/bes/graficos/0000000193cnt-s03criterios-reducibilidad.pdf.

17. Oakeshott $P$, Hunt GM. Long-term outcome in open spina bífida. Br J Gen Pract 2003;53(493):632-6.

18. Bol KA, Collins JS, Kirby RS. Survival of infants with neural tube defects in the presence of folic acid fortification. Pediatrics 2006;117(3):803-13.

19. Dalla Vecchia LK, Grosfeld JL, West KW, Rescorla FJ, et al. Intestinal atresia and stenosis: a 25-year experience with 277 cases. Arch Surg 1998;133(5):490-6.

20. Clark RH, Walker MW, Gauderer MW. Factors associated with mortality in neonates with gastroschisis. Eur J Pediatr Surg 2011;21(1):21-4.

21. Deng K, Qiu J, Dai L, Yi L, et al. Perinatal mortality in pregnancies with omphalocele: data from the Chinese national birth defects monitoring network, 1996-2006. BMC Pediatr 2014;14:160.

22. Alshehri A, Lo A, Baird R. An analysis of early nonmortality outcome prediction in esophageal atresia. J Pediatr Surg 2012;47(5):881-4.

23. García HJ, Aparicio de la Luz S, Franco-Gutiérrez M, González-Lara D, et al. Factores pronósticos asociados a mortalidad en recién nacidos con hernia diafragmática congénita. Gac Med Mex 2003;139(1):7-14.

24. TsaoK, Allison ND, Harting MT, Lally PA, et al. Congenital diaphragmatic hernia in the preterm infant. Surgery 2010;148(2):404-10.

25. Nembhard WN, Salemi JL, Ethen MK, Fixler DE, et al. Mortality among infants with birth defects: Joint effects of size at birth, gestational age, and maternal race/ethnicity. Birth Defects Res A Clin Mol Teratol 2010;88(9):728-36.

26. Hadidi A, Subotic U, Goeppl M, Waag KL. Early elective cesarean delivery before 36 weeks vs late spontaneous delivery in infants with gastroschisis. J Pediatr Surg 2008;43(7):1342-6.

27. Glasmeyer P, Grande C, Margarit J, Martí M, et al. Gastrosquisis. Cesárea electiva pretérmino y cierre primario inmediato; nuestra experiencia. Cir Pediatr 2012;25(1):12-5.

28. Carnaghan H, Pereira S, James CP, Charlesworth PB, et al. Is early delivery beneficial in gastroschisis? J Pediatr Surg 2014;49(6):928-33.

29. Maramreddy H, Fisher J, Slim M, Lagamma EF, et al. Delivery of gastroschisis patients before 37 weeks of gestation is associated with increased morbidities. J Pediatr Surg 2009;44(7):1360-6.

30. Adams-Chapman I, Hansen NI, Shankaran S, Bell EF, et al. Ten-year review of major birth defects in VLBW infants. Pediatrics 2013;132(1):49-61.

31. Okamoto T, Takamizawa S, Arai $\mathrm{H}$, Bitoh $\mathrm{Y}$, et al. Esophageal atresia: prognostic classification revisited. 
Surgery 2009;145(6):675-81.

32. Pawluk MS, Campaña H, Gili JA, Comas B, et al. Determinantes sociales adversos y riesgo para anomalías congénitas seleccionadas. Arch Argent Pediatr 2014; 112(3): 215-23.

33. Vrijheid M, Dolk H, Stone D, Abramsky L, et al. Socioeconomic inequalities in risk of congenital anomaly. Arch Dis Child 2000; 82(5):349-52.
34. Bronberg RA, Gutiérrez Redomero E, Alonso MC, Dipierri JE. Mortalidad infantil por malformaciones congénitas y condición socioeconómica: el caso de la Argentina. Rev Panam Salud Pública 2012; 31(6):469-75.

35. Smith LK, Budd JL, Field DJ, Draper ES. Socioeconomic inequalities in outcome of pregnancy and neonatal mortality associated with congenital anomalies: population based study. BMJ 2011;343:d4306.

\title{
Archivos hace 75 años
}

\author{
Hospital de Niños - Servicio de Infecciosas \\ Jefe: Prof. Dr. Florencio Bazán \\ Tratamiento de la erisipela del niño con las \\ sulfanilamidas ${ }^{*}$ ) \\ por los doctores \\ Florencio Bazán \\ Prof. adjunto de Clinica Pediátrica y \\ Puericultura \\ y \\ Docente libre de Clinica Pediátrica \\ Puericultura \\ El advenimiento de las sulfanilamidas y sus derivados en la \\ terapéutica anti-infecciosa, representa sin ninguna duda, una de \\ las adquisieiones más reales de la medicina moderna. \\ Desde el sensacional descubrimiento de Dornagk ( $\left.{ }^{1}\right)$, en 1935 , \\ los progresos sobre la nueva quimioterapia son sencillamente estu- \\ pendos. Las publicaciones a este respecto son numerosas y todos \\ los autores están concordes en afirmar los brillantes resultados ob- \\ tenidos con estos compuestos derivados de la sulfamidocrisoidina. \\ Es sobre todo en la erisipela que cabe señalar la evidente ac- \\ ción específica de la nueva medicación. El efecto sobre el estrep- \\ tococo y su mecanismo de acción han sido bien demostrados desde \\ los primeros estudios experimentales, corroborados éstos, luego, por \\ los hechos clínieos. \\ No entraremos en el detalle de estos estudios, sino simplemen- \\ te citaremos a los autores que más se han ocupado de esta cues- \\ tión. Merecen especial mención, los siguientes Levaditi y Vais- \\ man, $\left({ }^{2}\right)$, Tréfouël, Nitti y Bovet $\left({ }^{9}\right)$, Scheurer $\left({ }^{4}\right)$, Gmelin $\left({ }^{5}\right)$, \\ Colebrook y Kenny $\left({ }^{6}\right)$ Colebrook, Buttle y $\mathrm{O}^{\prime}$ Meara $\left({ }^{7}\right)$, Girard \\ $\left({ }^{8}\right)$, Marshall, Emerson y Cutting $\left({ }^{9}\right)$, Long y Bliss $\left({ }^{10}\right)$, Nitti y \\ Simón (11), Fourneau, Tréfouël, Nitti y Bovet $\left({ }^{12}\right)$, Rosenthal, \\ Bauer y Braham $\left({ }^{13}\right)$, Osgood y Brownlee $\left({ }^{14}\right)$, Fourneau $\left({ }^{15}\right)$, ete.
}




\section{ANEXO}

\section{BUENOS AIRES}

Hospital Abraham Piñeyro, Junín: Dra. Sonia Moretta, Dr. Jorge Herce.

Hospital Ana Goitía, Avellaneda: Dra. Adriana Mosquera, Dra. Valeria Vera.

Hospital Alende, Lomas de Zamora: Dr. Adrián Toffe, Dra. Ana Carolina Rocco.

Hospital Argentina Diego, Azul: Dra. Victoria Rizzo.

Hospital Bocalandro, Tres de Febrero: Dra. Mariana Oreglia, Dr. David Fernández.

Hospital Carlos Gianantonio, San Isidro: Dra. Blanca Cristina Senra, Dra. María Laura Sznitowsky.

Hospital Diego Thompson, San Martín: Dra. Alicia Fana, Dra. Lidia Santana.

Hospital Equiza, González Catán: Dr. Rubén Goncalvez, Dr. Rodolfo Zaiat.

Hospital Eurnekian, Ezeiza: Dr. José Vega Chancafe.

Hospital Eva Duarte de Perón (ex-Menem), Malvinas Argentinas: Dra. Elisa Duro.

Hospital Eva Perón, San Martín: Dra. Maricel Pontorno, Dra. Andrea Becerra.

Hospital Evita, Lanús: Dra. Marta Noemí Raggio, Dra. Graciela Schabvlosky.

Hospital Evita Pueblo, Berazategui: Dra. Mercedes Córdoba, Dr. Fabián Tomasoni.

Hospital Fiorito, Avellaneda: Dra. Felicitas Fumiere.

Hospital Gandulfo, Lomas de Zamora: Dr. Juan Carlos Delucca, Dra. Laura Botti,

Dra. Viviana Cosentino.

Hospital Héroes de Malvinas, Merlo: Dra. Patricia Wittbecker.

Hospital Iriarte, Quilmes: Dra. Graciela Carballido.

Hospital Larcade, San Miguel: Dra. Beatriz Velázquez, Dra. Fabiana Olivera.

Hospital Lucio Meléndez, Adrogué: Dra. Ana Barsellini, Dra. Ana Carolina Rocco.

Hospital M. V. Martínez, Pacheco: Dra. Stella Maris Benítez.

Hospital Mariano y Luciano de la Vega, Moreno: Dra. Adriana Dagostino, Dra. Sabrina Chattah.

Hospital Maternidad Santa Rosa, Vicente López: Dra. Carina Tula, Dra. Patricia Moreno.

Hospital Meissner, Pilar: Dra. Stella Maris Benítez, Dra. Natalia González.

Hospital Mercante, José C. Paz: Dra. Gabriela Klinge, Dra. Julieta Sada.

Hospital Mi Pueblo, Florencio Varela: Dra. Cecilia Iraira, Dra. María José Wernisch.

Hospital Narciso López, Lanús Este: Dra. Mónica Jewtuszyk, Dra. Viviana Pagani.

Hospital O. B. de Lavignolle, Morón: Dra. Teresa Gentile, Dra. Verónica Figueredo.

Hospital Oller, San Francisco Solano: Dr. Melvin Barrantes.

Hospital P. V. Cordero, San Fernando: Dr. Marcelo Prieto, Dra. María Marta Sánchez Vera.

Hospital Paroissien, La Matanza: Dra. Andrea Puss Barraza, Dra. Magdalena Bisbal.

Hospital Penna, Bahía Blanca: Dra. María Fernanda Maurín, Dra. Sonia Scardapane.

Hospital Nacional Alejandro Posadas, Haedo: Dra. Verónica Pingray, Dra. Isabel Micelli,

Lic. Alicia Aranaz.

Hospital Presidente Perón, Avellaneda: Dra. Mariana Brautigam.

Hospital San Felipe, San Nicolás: Dra. Graciela Olloco.

Hospital San José, Pergamino: Dra. Ángela Pacífico.

Hospital San Martín, La Plata: Dra. Graciela Ramos, Dr. Marcos Miró.

Hospital San Roque, La Plata: Lic. Noemí Orellano, Dra. Ana Ceccon, Dra. María Rosa Toncich.

Hospital Tetamanti, Mar del Plata: Dr. Eduardo Gil, Dr. Jorge Raverta.

Hospital Virgen del Carmen, Zárate: Dr. Carlos Bachiochi.

Hospital Emilia Ferreyra, Necochea: Dra. Liliana Espelet.

Hospital Dr. Ramón Santamarina, Tandil: Dr. Miguel Barrichelo.

\section{CABA}

Hospital Álvarez: Dra. M. Luisa Celadilla, Dra. Adriana Israilev.

Hospital Argerich: Dra. Ana Tocci, Dra. Karenith Santome.

Hospital Durand: Dr. Julio Falk, Dra. Noemí Nakayama.

Hospital Fernández: Dr. Ernesto Goldschmidt, Sra. Paula Molina.

Hospital Maternidad Sardá: Dra. Graciela Fernández, Sra. M. Carmen Arbones.

Hospital Penna: Dra. Elena Cristiano. 
Hospital Piñero: Dra. Mariana Panzitta, Dra. Gabriela Hernández, Dra. Claudia Epelbaun. Hospital Pirovano: Dr. Alberto Capriata.

Hospital Ramos Mejía: Dr. Rubén Bronberg.

Hospital Rivadavia: Dr. Luis Somaruga, Dra. Alcira Oliva.

Hospital Santojani: Dra. Daniela Rottenberg, Dra. Valeria Castellano.

Hospital Vélez Sársfield: Dr. Mario Mardyks.

Hospital de Clínicas José de San Martín: Dra. Estela Enriquez.

\section{CATAMARCA}

Maternidad 25 de Mayo: Dra. Inés Camacho, Lic. Marcos Toledo.

\section{CÓRDOBA}

Hospital Maternidad Nacional, Córdoba: Dra. Yanina Dalsasso, Dra. Marcela del Valle Ogas.

Hospital Maternidad Provincial, Córdoba: Dra. Cynthia Sánchez Topic,

Dra. Susana del Valle Ramaciotti.

Hospital Materno Neonatal, Córdoba: Dra. Raquel Lucía Torres, Dra. Marcela Beatriz Quaglia. Hospital Misericordia, Córdoba: Dra. Andrea Paola Chirino Misissian, Dra. Débora Gurevich. Hospital Pasteur, Villa María: Dra. Patricia Calvo.

Hospital Maternidad Kowalk, Río Cuarto: Dra. Diana Portela, Dra. Paola Pía Allende.

Hospital Río Cuarto, Río Cuarto: Dra. María Laura Bonora, Dra. Nydia Berguío.

\section{CORRIENTES}

Hospital J. Vidal, Corrientes: Dra. María del Rosario Córdoba, Lic. Elsa Aguirre.

Hospital Ángela Iglesias del Llano: Dra. Laura Palacios.

\section{CHACO}

Hospital J. Perrando, Resistencia: Dra. Dina Juárez de Ribles, Dra. Andrea Lew.

Hospital 4 de Junio, Pres. Roque Sáenz Peña: Dra. Ana Lucía Domínguez.

Hospital Güemes, Castelli: Dra. Rosana Medina.

\section{CHUBUT}

Hospital Andrés Isola, Pto. Madryn: Dra. María Soledad Silva, Lic. Norma Inés Segundo.

Hospital Centro Materno Infantil Trelew, Trelew: Dr. Raúl Musante.

Hospital Regional de Comodoro Rivadavia, Comodoro Rivadavia:

Dr. Maximiliano Medina Alarcón.

Hospital Zonal de Esquel, Esquel: Dr. Martín Batisttesa.

\section{ENTRE RÍOS}

Hospital Centenario, Gualeguaychú: Dra. Margarita Otaegui, Dr. Néstor Gieco.

Hospital Urquiza, Concepción del Uruguay: Dra. Adriana Gasparini, Dra. Adriana Reinoso.

Hospital Masvernat, Concordia: Dra. María Cecilia Arizabalo.

Hospital San Roque, Paraná: Lic. Mariel Bordenave.

\section{FORMOSA}

Hospital Madre y Niño, Formosa: Dra. Carina González, Dra. Marinela Antinori.

\section{JUJUY}

Hospital Guillermo Patterson, San Pedro: Dra. Mercedes Grau Toral.

Hospital Jorge Uro, La Quiaca: Dra. Nora Tolaba.

Hospital Orias, Libertador General San Martín: Dr. Alejandro Moragas.

Hospital Pablo Soria, San Salvador de Jujuy: Dra. Julia Flores, Dra. Claudia Carlos,

Dra. Sonia Alavar. 


\section{LA PAMPA}

Hospital Establecimiento Asistencial Gobernador Centeno, General Pico: Dr. Alejandro Irrazábal. Hospital Lucio Molas, Santa Rosa: Dra. Silvina Re.

\section{LA RIOJA}

Hospital de la Madre y el Niño (ex Vera Barros), La Rioja: Dra. Soledad Carrizo del Moral, Dra. Susana Beatriz Garello.

\section{MENDOZA}

Hospital Lagomaggiore, Mendoza: Dra. Cristina de Gaetano, Dra. Gabriela Torres.

Hospital Paroissien, Maipú: Dra. Claudia Beatriz Pizarro.

Hospital Perrupato, San Martín: Dra. Sonia Vilma Shiratori.

Hospital Scaravelli, Tunuyán: Dr. Gustavo Fernández.

Hospital Schestakow, San Rafael: Dr. Oscar Prieto.

\section{MISIONES}

Hospital El Dorado, El Dorado: Dra. Evelyn Heliana Spengler.

Hospital de Oberá, Oberá: Dra. Nelly Vaccari.

Hospital Ramón Madariaga, Posadas: Dra. Maris Carlucci, Dra. Bety Dedieu, Dra. Mónica Kosteki.

\section{NEUQUÉN}

Hospital Castro Rendón, Neuquén, Neuquén: Dra. María Eugenia Ponce Zaldúa, Dra. Silvia Avila. Hospital Heller, Neuquén: Dra. Silvia Avila, Dra. Luciana Fernández de Bon.

\section{RÍO NEGRO}

Hospital de General Roca, General Roca: Dra. Daniela Leimbgruber.

Hospital Ramón Carrillo, Bariloche: Dra. María Fabiana Tenreyro.

\section{SALTA}

Hospital Público Materno Infantil (ex Hospital El Milagro), Salta: Dra. Mariela Vilte, Dra. María Dolores Ruiz.

Hospital San Vicente Paul, San Ramón de la Nueva Orán: Dra. Alexandra Villareal, Dr. Franz Ancalle Michell.

Hospital Juan Domingo Perón, Tartagal: Dra. Beatriz Escalante.

\section{SAN JUAN}

Hospital Rawson, San Juan: Dra. Alejandra Sanz, Lic. Mirta Armesto, Dra. Silvia Mateos, Dra. Claudia Manrique.

\section{SAN LUIS}

Hospital Complejo Sanitario San Luis, San Luis: Dra. Laura Espinosa.

Hospital Juan Domingo Perón, Villa Mercedes: Dr. Jesús Ibáñez.

\section{SANTA CRUZ}

Hospital Regional de Río Gallegos, Río Gallegos: Dra. Alicia Susana Guanuco.

\section{SANTA FE}

Hospital Jaime Ferre, Rafaela: Dra. Miriam Estela Martínez.

Hospital Maternidad Martin, Rosario: Dra. Hilda Beatriz Fernández.

Hospital Provincial de Rosario, Rosario: Dra. Verónica Willimburgh.

Hospital Roque Sáenz Peña, Rosario: Dra. Griselda Arrastia, Dra. Gladis Terre.

Hospital Del Centenario, Rosario: Dra. Verónica Inés Cicao, Dr. Alejandro Rossi.

Hospital Eva Perón, Rosario: Dr. Fernando Basualdo, Dra. Natalia Vázquez Parachú.

Hospital Iturraspe, Santa Fe: Dra. Norma Domínguez. 
Hospital J. M. Cullen, Santa Fe: Dra. Laura Peralta.

Hospital Alejandro Gutiérrez, Venado Tuerto: Dr. Leonardo Andrés Fedre.

\section{SANTIAGO DEL ESTERO}

Hospital Ramón Carrillo, Santiago del Estero: Dra. Lidia Esther Padilla de Álvarez, Dra. Claudia Jugo, Dra. Mónica Morelli.

\section{TIERRA DEL FUEGO}

Hospital de Río Grande, Río Grande: Dr. Eduardo Zunino, Dra. Alejandra Albanesi.

Hospital de Ushuaia, Ushuaia: Dr. Sergio Nicolussi.

\section{TUCUMÁN}

Hospital Belascuain, Concepción: Dra. Lorena González Arias.

Hospital Maternidad Nuestra Señora de las Mercedes, S. M. de Tucumán: Dr. César Saleme.

Hospital Nicolás Avellaneda, S. M. de Tucumán: Dra. Claudia Montenegro. 